\title{
A Logistic Model Including Risk Factors for Lymph Node Metastasis Can Improve the Accuracy of Magnetic Resonance Imaging Diagnosis of Rectal Cancer
}

\author{
Shimpei Ogawa, Michio Itabashi*, Tomoichiro Hirosawa, Takuzo Hashimoto, \\ Yoshiko Bamba, Shingo Kameoka
}

\begin{abstract}
Background: To evaluate use of magnetic resonance imaging (MRI) and a logistic model including risk factors for lymph node metastasis for improved diagnosis. Materials and Methods: The subjects were 176 patients with rectal cancer who underwent preoperative MRI. The longest lymph node diameter was measured and a cut-off value for positive lymph node metastasis was established based on a receiver operating characteristic (ROC) curve. A logistic model was constructed based on MRI findings and risk factors for lymph node metastasis extracted from logistic-regression analysis. The diagnostic capabilities of MRI alone and those of the logistic model were compared using the area under the curve (AUC) of the ROC curve. Results: The cut-off value was a diameter of $5.47 \mathrm{~mm}$. Diagnosis using MRI had an accuracy of $65.9 \%$, sensitivity $73.5 \%$, specificity $61.3 \%$, positive predictive value (PPV) 62.9\%, and negative predictive value (NPV) 72.2\% [AUC: 0.6739 (95\% CI: 0.6016-0.7388)].Age (<59) $(p=0.0163), p T(T 3+T 4)(p=0.0001)$, and BMI $(<23.5)(p=0.0003)$ were extracted as independent risk factors for lymph node metastasis. Diagnosis using MRI with the logistic model had an accuracy of 75.0\%, sensitivity $72.3 \%$, specificity 77.4\%, PPV 74.1\%, and NPV 75.8\% [AUC: 0.7853 (95\% CI: 0.7098-0.8454)], showing a significantly improved diagnostic capacity using the logistic model $(\mathbf{p}=\mathbf{0 . 0 0 0 2})$. Conclusions: A logistic model including risk factors for lymph node metastasis can improve the accuracy of MRI diagnosis of rectal cancer.
\end{abstract}

Keywords: Rectal cancer - perirectal lymph nodes - lymph node metastasis - magnetic resonance imaging (MRI)

Asian Pac J Cancer Prev, 16 (2), 707-712

\section{Introduction}

Lymph node metastasis of rectal cancer in different stages had incidences of T1 11.9\%, T2 25.7\%, T3 55.9\%, and $\mathrm{T} 462.4 \%$ in a study of 6,442 patients in multiple centers in Japan (Watanabe et al., 2012). Thus, the incidence of lymph node metastasis of rectal cancer was very high in stage T3 and higher. Lymph node metastasis is a risk factor for local recurrence and a poor prognosis in rectal cancer (Quadros et al., 2012; Nadoshan et al., 2013) and the treatment strategy must account for lymph node metastasis (Colombo et al., 2011). Preoperative radiotherapy or chemoradiotherapy may reduce the risk of local recurrence (Sauer et al., 2004; Dou et al., 2013) and NCCN Guidelines recommend these therapies for rectal cancer with suspected lymph node metastasis (National Comprehensive Cancer Network., 2014).

Establishment of an indication for neoadjuvant therapy requires evaluation of nodal staging and $\mathrm{T}$ stage and mesorectal fascia (MRF) involvement. If the symptoms are underestimated due to inaccurate nodal staging, the patient may be judged to be ineligible for neoadjuvant therapy and may have a higher risk of local recurrence (White et al., 2013). In contrast, in a case in which the symptoms are overestimated, the patient may receive unnecessary adjuvant therapy that may lead to complications such as dysuria, dyschezia, and sexual dysfunction (Peeters et al., 2005; Guillem et al., 2008).

Diagnostic methods for lymph node metastasis of rectal cancer include endoscopic ultrasound (EUS), computed tomography (CT), and magnetic resonance imaging (MRI). These methods have accuracies of 61$80 \%, 56-79 \%$, and $57-85 \%$, respectively (Klessen et al., 2007), and a meta-analysis showed sensitivities and specificities of EUS, CT, and MRI of $67 \%$ and $78 \%$, $55 \%$ and $74 \%$, and $66 \%$ and $76 \%$, respectively (Bipat et al., 2004). Thus, none of these methods are currently satisfactory for diagnosis of lymph node metastasis of rectal cancer. However, combined imaging and a logistic model with risk factors from multivariate analysis may be an effective diagnostic approach (Garzotto et al., 2003; Xie et al., 2012). In this study, we evaluated whether use 
Shimpei Ogawa et al

of MRI and a logistic model of preoperative risk factors for lymph node metastasis can improve the accuracy of diagnosis of lymph node metastasis of rectal cancer.

\section{Materials and Methods}

\section{Patients}

The subjects were 176 consecutive patients with primary mid and low rectal cancer who underwent preoperative MRI at the Department of Surgery II, Tokyo Women's Medical University between January 1998 and December 2012. No patients received neoadjuvant therapy such as radiotherapy and chemotherapy (Table 1 ). The study was approved by the institutional review board and informed consent was obtained from all patients.

\section{MRI}

MRI was performed on a clinical $1.5 \mathrm{~T}$ system (Sigma, General Electric, Fairfield, CN, USA or Intera, Philips, Amsterdam, Netherlands) using T1-weighted (TR: 650 ms, TE: $10 \mathrm{~ms}$ ) and T2-weighted (TR: 4,600-4,800 $\mathrm{ms}$, TE: $100 \mathrm{~ms}$ ) imaging with and without contrast enhancement. The matrix size was $230 \times 512 \mathrm{~mm}$ for T1-weighted images and $282 \times 512 \mathrm{~mm}$ for T2-weighted images. The slice thickness was $5 \mathrm{~mm}$ and the inter-slice gap was $1 \mathrm{~mm}$. No bowel preparations, air insufflation or intravenous spasmolytic medications were used.

\section{MRI interpretation}

The longest diameters of perirectal lymph nodes (PRLNs) were measured using MRI. PRLNs located inside the MRF can be identified based on T1 hypointensity on MRI. Lymph nodes were detected on axial images by the authors or radiologists, and then measured by the authors. The longest diameter of the lymph nodes was measured on the images, and they were classified as MRIpositive (iN(+)) and MRI-negative (iN(-)) lymph nodes, as follows: $(i)$ all histologically negative lymph nodes in $(\mathrm{pN}(-))$ cases were included in the iN(-) group; (ii) for histologically metastasis-positive lymph nodes in $(\mathrm{pN}(+))$ cases, larger lymph nodes $(\mathrm{n}=\mathrm{b})$ were classified into the $\mathrm{iN}(+)$ group, and others were classified into the iN(-) group if the number of lymph nodes on an image (a) was greater than that of histologically positive lymph nodes (b) $(\mathrm{a} \geq \mathrm{b})$; and (iii) if the number of lymph nodes on an image (a) was smaller than that of histologically positive lymph nodes $(\mathrm{b})(\mathrm{a}<\mathrm{b})$, all lymph nodes were included in the iN(+) group. Lymph nodes classified into the iN(+) and iN(-) groups were matched with each longest diameter to make a receiver operating characteristic (ROC) curve. Maximum sensitivity and specificity were obtained from the ROC curve to give a diagnostic cut-off value based on the lymph node size.

\section{Diagnosis of lymph node metastasis using MRI}

The results of diagnostic imaging and histologic diagnosis were compared using the cut-off value. Of lymph nodes detected on images, the largest lymph node $\geq$ the cut-off value was classified into the $\mathrm{cN}(+)$ group and those with a size $<$ the cut-off value and those not detected on images were classified into the $\mathrm{cN}(-)$ group for comparison with the histologic diagnosis.

\section{Risk factors for lymph node metastasis}

Risk factors for lymph node metastasis were first identified in univariate logistic regression analysis of gender, age, location, macroscopic type, maximum tumor diameter, pathologic $\mathrm{T}$ stage (pT), histopathological grade, carcinoembryonic antigen (CEA) level, body mass index (BMI), and the presence of histological lymph node metastasis. Significant factors were subjected to multivariate analysis.

Predictive logistic model for lymph node metastasis

A logistic model was constructed for prediction of

Table 1. Clinicopathologic Characteristics of Patients

\begin{tabular}{|c|c|c|}
\hline Gender & $\begin{array}{l}\text { Male } \\
\text { Female }\end{array}$ & $\begin{array}{r}110 \\
66\end{array}$ \\
\hline \multicolumn{3}{|l|}{ Age (years) } \\
\hline & mean $\pm \mathrm{SD}$ (range) & $61.0 \pm 12.4(27-87)$ \\
\hline \multirow[t]{2}{*}{ Location } & Mid & 55 \\
\hline & Low & 121 \\
\hline \multicolumn{3}{|c|}{ Macroscopic type } \\
\hline & 0 & 4 \\
\hline & 1 & 17 \\
\hline & 2 & 141 \\
\hline & 3 & 8 \\
\hline & 4 & 6 \\
\hline \multicolumn{3}{|c|}{ Maximum tumor diameter (mm) } \\
\hline & mean $\pm \mathrm{SD}$ (range) & $48.0 \pm 20.5(10-140)$ \\
\hline \multicolumn{3}{|c|}{ Pathologic T stage } \\
\hline & $\mathrm{T} 1$ & 13 \\
\hline & $\mathrm{T} 2$ & 40 \\
\hline & $\mathrm{T} 3$ & 104 \\
\hline & $\mathrm{T} 4$ & 19 \\
\hline \multicolumn{3}{|c|}{ Histopathological grade } \\
\hline & G1 & 69 \\
\hline & $\mathrm{G} 2$ & 93 \\
\hline & G3 & 5 \\
\hline & G4 & 9 \\
\hline \multicolumn{3}{|c|}{ Lymphatic invasion } \\
\hline & L0 & 28 \\
\hline & L1 & 148 \\
\hline \multicolumn{3}{|c|}{ Venous invasion } \\
\hline & V0 & 105 \\
\hline & V1 & 71 \\
\hline \multicolumn{3}{|c|}{ Pathologic N stage } \\
\hline & No & 93 \\
\hline & N1 & 53 \\
\hline & $\mathrm{N} 2$ & 30 \\
\hline \multirow[t]{2}{*}{ CEA } & $<5$ & 110 \\
\hline & $\geq 5$ & 66 \\
\hline \multirow[t]{2}{*}{ BMI } & $<23.5$ & 115 \\
\hline & $\geq 23.5$ & 61 \\
\hline \multicolumn{3}{|c|}{ Surgical procedure } \\
\hline & Anterior resection & 119 \\
\hline & Abdominoperineal resection & 41 \\
\hline & Hartmann operation & 3 \\
\hline & Intersphincteric resection & 13 \\
\hline \multicolumn{3}{|c|}{ Number of retrieved lymph nodes } \\
\hline & mean $\pm \mathrm{SD}$ (range) & $17.3 \pm 13.2(1-81)$ \\
\hline \multirow[t]{4}{*}{ TNM stage } & I & 40 \\
\hline & II & 45 \\
\hline & III & 65 \\
\hline & IV & 26 \\
\hline
\end{tabular}

*CEA, carcinoembryonic antigen; BMI, body mass index 
Logistic Model for Lymph Node Metastasis Can Improve the Accuracy of MRI Diagnosis of Rectal Cancer

lymph node metastasis (Np) using the MRI diagnosis and the extracted risk factors as explanatory variables. The standard logistic regression formula Logit $(\mathrm{Np})=\beta_{0}+\beta_{1} X_{1}$ $+\beta_{2} X_{2}+\ldots+\beta n X n$ was used, where Logit $(\mathrm{Np})=\ln [\mathrm{Np} /(1-$ $\mathrm{Np})$ ], and " $n$ " is the number of influence factors, " $\beta$ " is the influence coefficient, " $X$ " is the influence factor, and " $\beta_{0}$ " is a constant. Np values of $\geq 0.5$ and $<0.5$ calculated from the logistic model were used to classify cases as positive $(\mathrm{N}(+))$ and negative $(\mathrm{lN}(-))$ for lymph node metastasis, respectively, for comparison with the histologic diagnosis. The diagnostic capability of MRI alone and that with the logistic model were also compared using the area under the curve (AUC) of the ROC curve.

\section{Statistical analysis}

Statistical analysis was performed using ROC analysis, a chi-square test for independence, a Fisher exact probability test, and multivariate logistic regression analysis. In all tests, $\mathrm{p}<0.05$ was considered to indicate a significant difference. All analyses were performed using JMP ver. 10.0.2 for Windows $\left({ }^{\circledR}\right)$ (SAS Institute Inc., Cary, NC, USA).

\section{Results}

Diagnosis of lymph node metastasis using MRI

A total of 510 lymph nodes were identified in 144 of 176 subjects. No lymph nodes were identified in 32 subjects. Of the 510 lymph nodes, 173 and 337 were $\mathrm{iN}(+)$ and $\mathrm{iN}(-)$, respectively. The mean size in the $\mathrm{iN}(+)$ group $(7.27 \pm 3.45 \mathrm{~mm})$ was significantly greater than that in the iN(-) group $(4.79 \pm 2.39 \mathrm{~mm})$. Comparison with histological diagnoses using a cut-off value of $5.47 \mathrm{~mm}$ obtained from the ROC curve gave an accuracy of $65.9 \%$, sensitivity $73.5 \%$, specificity $61.3 \%$, PPV $62.9 \%$, and NPV 72.2\% for diagnostic imaging (Table 2).

\section{Risk factors for lymph node metastasis}

Univariate analysis identified age $(\mathrm{p}=0.0132)$, macroscopic type $(\mathrm{p}=0.0228)$, maximum tumor diameter $(\mathrm{p}=0.0018), \mathrm{pT}(\mathrm{p}<0.0001)$, CEA $(\mathrm{p}=0.0140)$, and BMI $(\mathrm{p}=0.0006)$ as significant risk factors for lymph node metastasis. In multivariate logistic regression analysis, age $(<59)(\mathrm{p}=0.0163), \mathrm{pT}(\mathrm{T} 3+\mathrm{T} 4)(\mathrm{p}=0.0001)$, and BMI $(<23.5)(\mathrm{p}=0.0003)$ were extracted as independent risk factors for lymph node metastasis (Table 3 ).

\section{Predictive logistic model for lymph node metastasis}

The logistic model containing age, pT, BMI, and MRI diagnosis was as follows: Logit $(\mathrm{Np})=-0.6966+$ $0.4062 \mathrm{X}_{1}+(-0.3690) \mathrm{X}_{2}+(-0.8176) \mathrm{X}_{3}+(-0.6260) \mathrm{X}_{4} \mathrm{X}_{1}$ : [cN(+): 1,cN(-): -1], $\mathrm{X}_{2}$ : [Age $\geq 59: 1$, Age $\left.<59:-1\right], X 3:$ : [pT T1+T2: 1, pT T3+T4: -1], $\mathrm{X}_{4}$ : [BMI $\geq 23.5: 1, \mathrm{BMI}<23.5$ : -1] (Table 4). An analysis using this model resulted in

Table 2. Comparison between MRI Diagnosis and Logistic Model Diagnosis

\begin{tabular}{|c|c|c|c|c|c|c|c|}
\hline & \multicolumn{2}{|c|}{ Histological diagnosis } & \multicolumn{5}{|c|}{ Diagnostic Value (\%) } \\
\hline & Yes & No & Accuracy & Sensitivity & Specificity & PPV & NPV \\
\hline MRI & & & 65.9 & 73.5 & 61.3 & 62.9 & 72.2 \\
\hline $\mathrm{cN}(+)^{\dagger}$ & 61 & 36 & & & & & \\
\hline $\mathrm{cN}(-)^{\dagger}$ & 22 & 57 & & & & & \\
\hline Logistic model & & & 75.0 & 72.3 & 77.4 & 74.1 & 75.8 \\
\hline $1 \mathrm{~N}(+)^{\ddagger}$ & 60 & 21 & & & & & \\
\hline $1 \mathrm{~N}(-)^{\ddagger}$ & 23 & 72 & & & & & \\
\hline
\end{tabular}

MRI, magnetic resonance imaging; PPV, positive predictive value; NPV, negative predictive value; ${ }^{\dagger}$ Diagnosis of lymph node metastasis using magnetic resonance imaging; Diagnosis of lymph node metastasis using logistic model

Table 3. Logistic Regression Analysis of Risk Factors for Lymph-node Metastasis

\begin{tabular}{|c|c|c|c|c|c|c|}
\hline & & Positive & Negative & Univariate analysis & Multiv & ate analysis \\
\hline & & $\mathrm{n}=83(\%)$ & $\mathrm{n}=93(\%)$ & $\mathrm{p}$ value & $\mathrm{p}$ value & OR(95\%CI) \\
\hline Gender & Male & $51(61.4)$ & $59(63.4)$ & 0.7849 & & \\
\hline & Female & $32(38.6)$ & $34(36.6)$ & & & \\
\hline Age (years) & $<59$ & $43(51.8)$ & $31(33.3)$ & 0.0132 & 0.0163 & $2.35(1.17-4.81)$ \\
\hline & $\geq 59$ & $40(48.2)$ & $62(66.7)$ & & & \\
\hline Location & Mid & $26(31.3)$ & $29(31.2)$ & 0.9838 & & \\
\hline & Low & $57(68.7)$ & $64(68.8)$ & & & \\
\hline Macroscopic type & $0,1,2$ & $72(86.7)$ & $90(96.8)$ & 0.0228 & 0.0602 & $3.66(0.95-18.6)$ \\
\hline & 3,4 & $11(13.3)$ & $3(3.2)$ & & & \\
\hline Maximum tumor diameter & $<48 \mathrm{~mm}$ & $34(41.0)$ & $60(64.5)$ & 0.0018 & 0.5600 & $1.25(0.59-2.61)$ \\
\hline & $\geq 48 \mathrm{~mm}$ & $49(59.0)$ & $33(35.5)$ & & & \\
\hline Pathologic T stage & $\mathrm{T} 1, \mathrm{~T} 2$ & $10(12.0)$ & $43(46.2)$ & $<0.0001$ & 0.0001 & $5.45(2.26-14.1)$ \\
\hline & $\mathrm{T} 3, \mathrm{~T} 4$ & $73(88.0)$ & $50(53.8)$ & & & \\
\hline Histopathological grade & $\mathrm{G} 1, \mathrm{G} 2$ & 76(91.6) & $86(92.5)$ & 0.8244 & & \\
\hline & $\mathrm{G} 3, \mathrm{G} 4$ & $7(8.4)$ & $7(7.5)$ & & & \\
\hline CEA & $<5$ & $44(53.0)$ & $66(71.0)$ & 0.0140 & 0.2998 & $1.49(0.70-3.19)$ \\
\hline & $\geq 5$ & $39(47.0)$ & $27(29.0)$ & & & \\
\hline BMI & $<23.5$ & $65(78.3)$ & $50(53.8)$ & 0.0006 & 0.0003 & $3.87(1.85-8.46)$ \\
\hline & $\geq 23.5$ & $18(21.7)$ & $43(46.2)$ & & & \\
\hline
\end{tabular}


Table 4. Parameter Estimates for the Logistic Model for Lymph Node Metastasis

\begin{tabular}{|c|c|c|c|c|c|c|c|}
\hline \multirow[t]{2}{*}{ Variable } & \multirow[t]{2}{*}{ Influence factor } & \multirow[t]{2}{*}{$\beta$} & \multirow[t]{2}{*}{ S.E } & \multirow[t]{2}{*}{$\chi^{2}$} & \multirow[t]{2}{*}{$\mathrm{p}$} & \multicolumn{2}{|c|}{$95 \% \mathrm{CI}$} \\
\hline & & & & & & Lower & Upper \\
\hline $\mathrm{X} 1$ & $\mathrm{cN}^{\dagger}$ & 0.4062 & 0.1872 & 4.71 & 0.0300 & 0.0384 & 0.7753 \\
\hline $\mathrm{X} 2$ & Age & -0.3690 & 0.1792 & 4.24 & 0.0395 & -0.7260 & -0.0206 \\
\hline $\mathrm{X} 3$ & Pathologic T stage & -0.8176 & 0.2217 & 13.60 & 0.0002 & -1.2729 & -0.3973 \\
\hline $\mathrm{X} 4$ & $\begin{array}{c}\text { BMI } \\
\text { constant }\end{array}$ & $\begin{array}{l}-0.6260 \\
-0.6966\end{array}$ & 0.1937 & 10.45 & 0.0012 & -1.0163 & -0.2539 \\
\hline
\end{tabular}

BMI, Body Mass Index; ${ }^{\dagger}$ Diagnosis of lymph node metastasis using magnetic resonance imaging

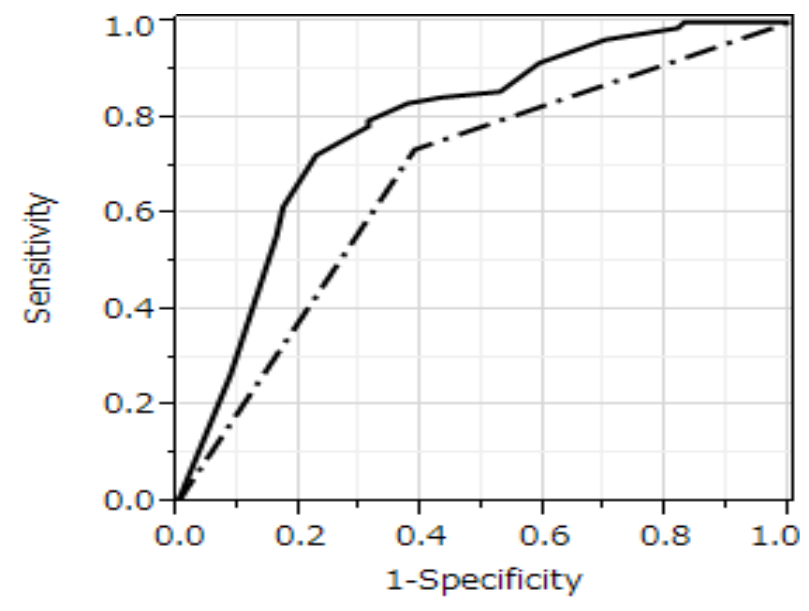

Figure 1.AUCs Obtained from Each ROC Curve using MRI Alone (Dashed Line) and from Diagnosis using MRI and the Logistic Model (Full Line) were 0.6739 (95\% CI: 0.6016-0.7388) and 0.7853 (95\% CI: 0.70980.8454), Respectively. The AUCs differed Significantly between the Two Approaches $(p=0.0002)$

$1 \mathrm{~N}(+)$ and $1 \mathrm{~N}(-)$ groups of 81 and 95 subjects, respectively. Comparison with histological diagnoses gave an accuracy, sensitivity, specificity, PPV, and NPV of $75.0 \%, 72.3 \%$, $77.4 \%, 74.1 \%$, and $75.8 \%$, respectively (Table 2 ). The AUC obtained from ROC curves for MRI alone and diagnosis with the logistic model were 0.6739 (95\% CI: 0.6016-0.7388) and 0.7853 (95\% CI: 0.7098-0.8454), respectively, with a significant difference between these AUC values ( $p=0.0002)$ (Figure 1).

\section{Discussion}

MRI has high soft-tissue contrast resolution and multiplanar imaging capacity, and thus is widely used in preoperative staging of rectal cancer (Raghunathan et al., 2009). In particular, MRI is the most reliable method for evaluating T factors and the MRF. ESMO Guidelines advise use of EUS for T1 staging, but recommend MRI as the first choice for location, T stage (T2/T3/T4), sphincter infiltration, and MRF involvement (Schmoll et al., 2012). MRI can also examine the inner and outer mesorectum for $\mathrm{N}$ staging. However, a meta-analysis found a lower diagnostic odds ratio with MRI for rectal cancer lymph node metastasis compared to diagnosis of $\mathrm{T}$ stage and MRF involvement, indicating that MRI cannot evaluate lymph nodes accurately and is insufficient for complete diagnosis (Al-Sukhni et al., 2012).

One problem with current MRI diagnosis is the absence of clear diagnostic criteria. The widely accepted
MRI diagnostic criterion for lymph node metastasis is the size of the lymph nodes on images (Brown et al., 2003; Ogawa et al., 2014). This is based on the finding that a lymph node positive for metastasis is usually bigger than a negative lymph node, but small lymph nodes are also often positive. Brown et al. found that $45.5 \%$ of $>5$ $\mathrm{mm}$ lymph nodes were metastatic, but $15.3 \%$ of $\leq 5-\mathrm{mm}$ lymph nodes were also positive for metastasis (Brown et al., 2003). Positive and negative lymph nodes have also been shown to largely overlap in a histogram of the maximum diameter. Thus, it is difficult to define a precise cut-off value based on the size of a metastasis-positive lymph node. Morphological diagnostic criteria such as signal heterogeneity and an irregular border can also be used (Brown et al., 2003), but a lymph node of $\leq 4 \mathrm{~mm}$ may not be correctly diagnosed based on irregularities or heterogeneous signals (Akasu et al., 2009). Thus, morphological diagnostic criteria cannot be used for small lymph nodes. Current high-resolution MRI can visualize a 2-mm lymph node, but cannot show whether it is benign or malignant. A size of $\geq 5 \mathrm{~mm}$ may be needed to permit evaluation of the morphology, margin, and signal intensity (Beets-Tan et al., 2011).

These limitations of current MRI technology for correct diagnosis of lymph node metastasis led us to evaluate use of a combination of MRI and a logistic model including risk factors for lymph node metastasis. Diagnosis of lymph node metastasis using a combination of risk factors and imaging findings, such as CT and diagnosis from a logistic model with multiple risk factors, has been shown to be viable (Wang et al., 2012; Stiekema et al., 2014). Thus, Wang et al. showed that a combination of serum vascular endothelial growth factor-C (SVEGF-C) levels with a CT scan can improve the accuracy of diagnosis of lymph node metastasis in gastric cancer (Wang et al., 2012).

Many studies of lymph node metastasis of rectal cancer have been conducted in T1/T2 cases, and T-stage and sm-level, infiltration of nerves and vessels, lymphatic invasion, histologic differentiation, and tumor size and tumor location have been identified as risk factors (Chang et al., 2012; Saraste et al., 2013). However, most of these factors can only be determined in postoperative histopathological analysis. It is ideal if the prediction of lymph node metastasis by preoperative factors (Gao et al., 2013). In the current study, preoperative factors of age $(<59)$ and BMI $(<23.5)$, and predictable to some extent pT (T3+T4) were extracted as independent risk factors for lymph node metastasis. A meta-analysis showed that MRI has good accuracy for T category and should be considered for preoperative rectal cancer (Al-Sukhni et al., 2012). It seems to be possible to relatively accurately discrimination 
of $\mathrm{T} 1+\mathrm{T} 2$ or $\mathrm{T} 3+\mathrm{T} 4$. By adding these factors to the MRI diagnosis, a logistic model to predict lymph node metastasis was constructed. Using $1 \mathrm{~N}(+)$ and $1 \mathrm{~N}(-)$ groups based on prediction of lymph node metastasis using the logistic model, comparison with histologic diagnoses gave AUCs of 0.6739 for MRI alone and 0.7853 for the logistic model, indicating significant improvement of diagnostic capability using the logistic model.

Diagnosis of lymph node metastasis using imaging is a direct method that depends on visualization of lymph nodes to determine whether the lymph nodes are involved in metastasis. Thus, the diagnostic capability may vary depending on the equipment and more accurate diagnosis may be produced by development of new equipment and contrast media. However, the current method of diagnosis of lymph node metastasis using imaging alone is clearly limited. The current study shows that inclusion of indirect findings of risk factors for lymph node metastasis can improve the diagnostic accuracy. We note that the study was performed in a single facility with a small number of subjects, and the findings needs to be evaluated through data accumulation and a future prospective study. Furthermore, it is also possible that diagnosis with a combination of other risk factors for lymph node metastasis may also improve the diagnostic performance, and a study of multiple factors is required. Within these limitations, we suggest that improved staging using the logistic model will allow appropriate preoperative radiotherapy and chemoradiotherapy to be performed in eligible patients. Thus, accurate diagnosis of rectal cancer with lymph node metastasis is likely to lead to optimal treatment that will decrease complications and improve outcomes.

\section{Acknowledgements}

We did not receive financial support or compensation related to the development of the manuscript.

\section{References}

Akasu T, Iinuma G, Takawa M, et al (2009). Accuracy of highresolution magnetic resonance imaging in preoperative staging of rectal cancer. Ann Surg Oncol, 16, 2787-94.

Al-Sukhni E, Milot L, Fruitman M, et al (2012). Diagnostic accuracy of MRI for assessment of T category, lymph node metastases, and circumferential resection margin involvement in patients with rectal cancer: a systematic review and meta-analysis. Ann Surg Oncol, 19, 2212-23.

Beets-Tan RG, Beets GL (2011). Local staging of rectal cancer: a review of imaging. J Magn Reson Imaging, 33, 1012-9.

Bipat S, Glas AS, Slors FJ, et al (2004). Rectal cancer: local staging and assessment of lymph node involvement with endoluminal US, CT, and MR imaging-a meta-analysis. Radiology, 232, 773-83.

Brown G, Richards CJ, Bourne MW, et al (2003). Morphologic predictors of lymph node status in rectal cancer with use of high-spatial-resolution MR imaging with histopathologic comparison. Radiology, 227, 371-7.

Chang HC, Huang SC, Chen JS, et al (2012). Risk factors for lymph node metastasis in pT1 and pT2 rectal cancer: a single-institute experience in 943 patients and literature review. Ann Surg Oncol, 19, 2477-84.
Colombo PE, Patani N, Bibeau F, et al (2011). Clinical impact of lymph node status in rectal cancer. Surg Oncol, 20, 227-33.

Dou X, Wang RB, Yan HJ, et al (2013). Circulating lymphocytes as predictors of sensitivity to preoperative chemoradiotherapy in rectal cancer cases. Asian Pac J Cancer Prev, 14, 3881-5.

Gao C, Li JT, Fang L, et al (2013). Pre-operative predictive factors for intra-operative pathological lymph node metastasis in rectal cancers. Asian Pac J Cancer Prev, 14, 6293-9.

Garzotto M, Hudson RG, Peters L, et al (2003). Predictive modeling for the presence of prostate carcinoma using clinical, laboratory, and ultrasound parameters in patients with prostate specific antigen levels $<$ or $=10 \mathrm{ng} / \mathrm{mL}$. Cancer, 98, 1417-22.

Guillem JG, Diaz-Gonzalez JA, Minsky BD, et al (2008). cT3N0 rectal cancer: potential overtreatment with preoperative chemoradiotherapy is warranted. J Clin Oncol, 26, 368-73.

Klessen C, Rogalla P, Taupitz M (2007). Local staging of rectal cancer: the current role of MRI. Eur Radiol, 17, 379-89.

Nadoshan JJ, Omranipour R, Beiki O, et al (2013). Prognostic value of lymph node ratios in node positive rectal cancer treated with preoperative chemoradiation. Asian Pac J Cancer Prev, 14, 3769-72.

National Comprehensive Cancer Network. NCCN clinical practice guidelines in oncology (NCCN Guidelines TM), Rectal Cancer. Version 1. 2015 [cited 1 November 2014].

Ogawa S, Itabashi M, Hirosawa T, et al (2014). Lateral pelvic lymph node dissection can be omitted in lower rectal cancer in which the longest lateral pelvic and perirectal lymph node is less than $5 \mathrm{~mm}$ on MRI. J Surg Oncol, 109, 227-33.

Peeters KC, van de Velde CJ, Leer JW, et al (2005). Late side effects of short-course preoperative radiotherapy combined with total mesorectal excision for rectal cancer: increased bowel dysfunction in irradiated patients-a Dutch colorectal cancer group study. J Clin Oncol, 23, 6199-206.

Quadros CA, Falcao MF, Carvalho ME, Ladeia PA, Lopes A (2012). Metastases to retroperitoneal or lateral pelvic lymph nodes indicated unfavorable survival and high pelvic recurrence rates in a cohort of 102 patients with low rectal adenocarcinoma. J Surg Oncol, 106, 653-8.

Raghunathan G, Mortele KJ (2009). Magnetic resonance imaging of anorectal neoplasms. Clin Gastroenterol Hepatol, 7, 379-88.

Saraste D, Gunnarsson U, Janson M (2013). Predicting lymph node metastases in early rectal cancer. Eur J Cancer, $\mathbf{4 9}$, 1104-8.

Sauer R, Becker H, Hohenberger W, et al (2004). Preoperative versus postoperative chemoradiotherapy for rectal cancer. N Engl J Med, 351, 1731-40.

Schmoll HJ, Van Cutsem E, Stein A, et al (2012). ESMO Consensus Guidelines for management of patients with colon and rectal cancer. a personalized approach to clinical decision making. Ann Oncol, 23, 2479-516.

Stiekema A, Lok CA, Kenter GG, et al (2014). A predictive model combining human epididymal protein 4 and radiologic features for the diagnosis of ovarian cancer. Gynecol Oncol, 132, 573-7.

Wang TB, Wang J, Wei XQ, Wei B, Dong WG (2012). Serum vascular endothelial growth factor-C combined with multidetector CT in the preoperative diagnosis of lymph node metastasis of gastric cancer. Asia Pac J Clin Oncol, 8, 180-6.

Watanabe T, Itabashi M, Shimada Y, et al (2012). Japanese society for cancer of the colon and rectum (JSCCR) guidelines 2010 for the treatment of colorectal cancer. Int $J$ Clin Oncol, 17, 1-29.

White R, Ung KA, Mathlum M (2013). Accuracy of magnetic resonance imaging in the pre-operative staging of rectal 
Shimpei Ogawa et al

adenocarcinoma: Experience from a regional Australian cancer center. Asia Pac J Clin Oncol, 9, 318-23.

Xie F, Yang H, Wang S, et al (2012). A logistic regression model for predicting axillary lymph node metastases in early breast carcinoma patients. Sensors, 12, 9936-50. 